Vantage: Journal of Thematic Analysis

ISSN: 2582-7391

A Multidisciplinary Publication of Centre for Research,

Maitreyi College, University of Delhi

April 2021, Volume 2, Issue 1

Invited Article

\title{
What does Our Experience of COVID-19 Teach Us?
}

\author{
Shivcharan Prasad and Ipsita Roy* \\ Department of Biotechnology, National Institute of Pharmaceutical Education and \\ Research Sector 67, S.A.S. Nagar, Punjab-160062, India \\ *Correspondence: ipsita@niper.ac.in
}

\section{INTRODUCTION}

Viral diseases pose the greatest concern for human health. Some of the major epidemics have turned into pandemics, such as severe acute respiratory syndrome (SARS), Middle East respiratory syndrome (MERS), HIV, influenza A (H1N1) $\mathrm{pdm} / 09$, and most recently, COVID-19. It soon became a major burden for all affected countries, which disturbed social and economic development. As of March 5, 2021, there have been over 116 million confirmed COVID-19 cases and over 2.5 million deaths reported worldwide (https://www.worldometers.info/coronavirus/, accessed on 05.03.2021). COVID-19 could cost the global economy about USD 4.1 trillion (https://cepi.net/about/whyweexist/; accessed on 05.03.2021). This is not the first time that humans have encountered coronavirus; several other zoonotic outbreaks of coronavirus have been reported earlier. Among these was the SARS-CoV (Severe Acute Respiratory Syndrome coronavirus) outbreak in 2003 in some parts of China which later spread to a few other countries. There were around 8000 confirmed cases and 774 reported deaths (Lundstrom, 2020). Another outbreak of coronavirus occurred in Saudi Arabia and other Middle Eastern countries in 2012 and was named as MERS-CoV (Middle East Respiratory Syndrome coronavirus) (Al-Osail \& AlWazzah, 2017). Although the disease did not exhibit a widespread outbreak, it resulted in a very high fatality rate, with 855 cases and 333 deaths (Lundstrom, 2020). 
COVID-19, on the other hand, has affected almost all the countries or territories around the globe. Statistics for some of the most affected countries is shown in Fig 1.

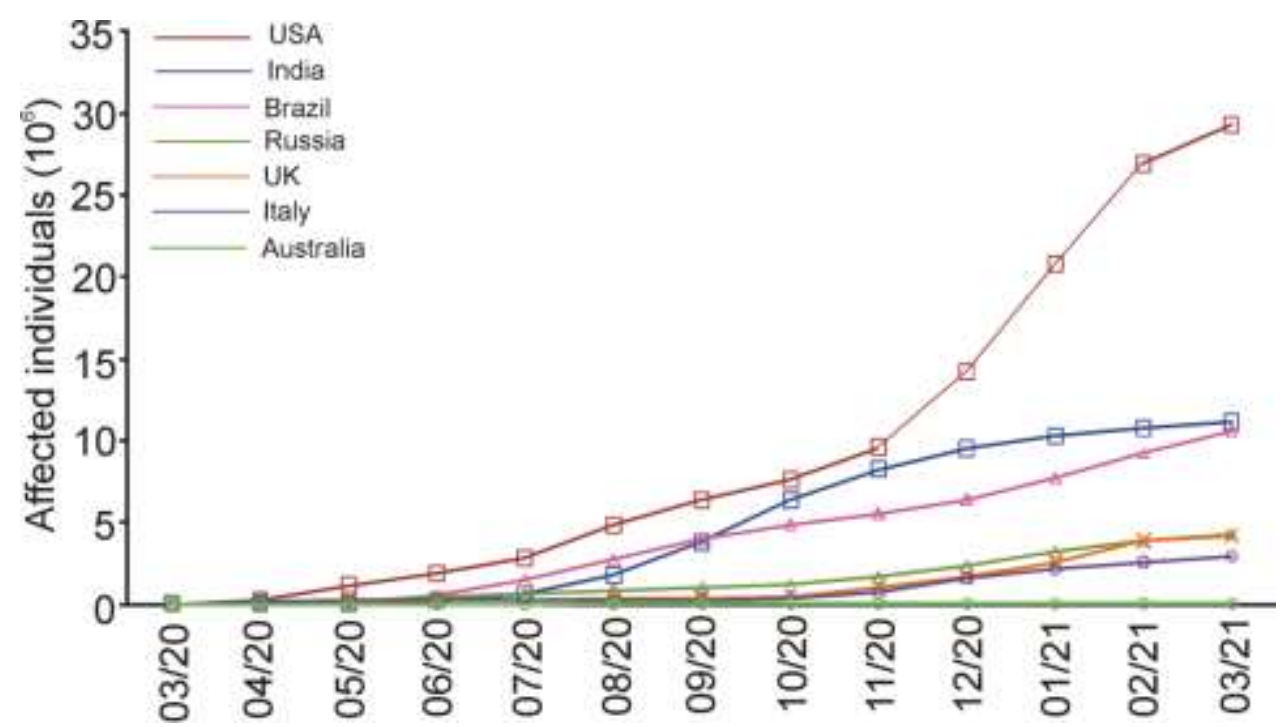

Figure 1: Some of the countries affected by COVID-19, data shown on the first of each month (https://www.worldometers.info/coronavirus/, accessed on 05.03.2021).

The focus of this mini review is on the experience gained by different sectors during this challenging time and how that experience can be utilized to ensure a better response to the next public health threat.

\section{BIOLOGY OF CORONAVIRUS}

Coronaviruses are a class of enveloped positive-sense single-stranded RNA viruses that cause respiratory symptoms and sometimes, enteric, hepatic or neuronal disorders (Masters, 2006). Coronaviruses are members of the family Coronaviridae and subfamily Orthocoronavirinae. The subfamily is further divided into alphacoronaviruses and betacoronaviruses which infect mammals, while gammacoronaviruses and deltacoronaviruses can infect birds too. The avian infectious bronchitis virus, which causes respiratory disease with low fatality in poultry but not other birds, is a gammacoronavirus. Like all other RNA viruses which infect eukaryotic cells, the genome of coronavirus shows capping at the $5^{\prime}$ and $3^{\prime}$ untranslated regions which allows it to utilize the machinery of the host cells for its replication. The genome of coronavirus is also unusually large as compared to other RNA viruses. It varies between $26-32 \mathrm{~kb}$ (Woo et al., 2010) and contains multiple ORFs. Compared to this, the genome size of the 
poliovirus, another positive strand RNA virus is $~ 7500$ nucleotides (Tavares et al., 2013) while the genome size of the segmented influenza A virus is 13,588 nucleotides (Ghedin et al., 2005). Larger genome sizes have been inversely correlated with mutation rates which may affect their adaptation to hosts (Lauber et al., 2013).

Infection by coronaviruses which use humans as hosts, e.g. HCoV-229E, HCoVOC43, HCoV-NL63 and HCoV-HKU1, can be acute or chronic, and usually cause mild symptoms associated with 'common cold'. However, the recently emerged members, viz. SARS-CoV, MERS-CoV and SARS-CoV-2, have far deadlier consequences with some of them showing significant fatality rates. Therapeutic strategies targeting SARS-CoV-2 concentrate on both prophylactic (Table 1) and curative (Table 2) aspects, with variable results.

Table 1: List of vaccines against SARS-CoV-2 which are approved and are under development [https://www.raps.org/news-and-articles/news-articles/2020/3/covid-19-vaccinetracker, https://covid19.trackvaccines.org/vaccines/]

\begin{tabular}{|l|l|l|l|l|}
\hline \multicolumn{5}{|c|}{ Authorized/Approved vaccine } \\
\hline Name & Vaccine type & Primary developer & $\begin{array}{l}\text { Country of } \\
\text { origin }\end{array}$ & $\begin{array}{l}\text { Authorization/ } \\
\text { Approval }\end{array}$ \\
\hline $\begin{array}{l}\text { Comirnaty } \\
\text { BNT162b2) }\end{array}$ & $\begin{array}{l}\text { mRNA-based } \\
\text { vaccine }\end{array}$ & $\begin{array}{l}\text { Pfizer, BioNTech; Fosun } \\
\text { Pharma }\end{array}$ & Multinational & $\begin{array}{l}\text { Approved in 63 } \\
\text { countries, } \\
9 \text { trials in 9 } \\
\text { countries }\end{array}$ \\
\hline $\begin{array}{l}\text { Moderna } \\
\text { COVID-19 Vaccine } \\
\text { (mRNA-1273) }\end{array}$ & $\begin{array}{l}\text { mRNA-based } \\
\text { vaccine }\end{array}$ & Moderna, BARDA, NIAID & USA & $\begin{array}{l}\text { Approved in 40 } \\
\text { countries, } \\
\text { trials in 1 } \\
\text { country (Phase } \\
3 \text { clinical trials) }\end{array}$ \\
\hline $\begin{array}{l}\text { COVID-19 Vaccine } \\
\text { AstraZeneca } \\
\text { AZD1222); also } \\
\text { known as Covishield }\end{array}$ & $\begin{array}{l}\text { Adenovirus } \\
\text { vaccine }\end{array}$ & BARDA, OWS & UK & $\begin{array}{l}\text { Approved in 53 } \\
\text { countries, } \\
18 \text { trials in 13 } \\
\text { countries } \\
\text { (Phase 3 } \\
\text { clinical trials) }\end{array}$ \\
\hline Sputnik V & $\begin{array}{l}\text { Non- } \\
\text { replicating } \\
\text { viral vector }\end{array}$ & $\begin{array}{l}\text { Gamaleya Research } \\
\text { Institute, Acellena Contract } \\
\text { Drug Research and } \\
\text { Development }\end{array}$ & $\begin{array}{l}\text { Russia } \\
\text { vaccine } \\
\text { formalin } \\
\text { with alum } \\
\text { adjuvant) }\end{array}$ & $\begin{array}{l}\text { Sinovac } \\
\text { Approved in 29 } \\
\text { countries, } \\
15 \text { trials in 6 } \\
\text { countries }\end{array}$ \\
\hline CoronaVac & $\begin{array}{l}\text { Inactivated } \\
\text { countries, } \\
11 \text { trials in 5 } \\
\text { countries }\end{array}$ \\
\hline
\end{tabular}




\begin{tabular}{|c|c|c|c|c|}
\hline \multicolumn{5}{|c|}{ Authorized/Approved vaccine } \\
\hline Name & Vaccine type & Primary developer & $\begin{array}{l}\text { Country of } \\
\text { origin }\end{array}$ & $\begin{array}{l}\text { Authorization/ } \\
\text { Approval }\end{array}$ \\
\hline BBIBP-CorV & $\begin{array}{l}\text { Inactivated } \\
\text { vaccine }\end{array}$ & $\begin{array}{l}\text { Beijing Institute of } \\
\text { Biological Products; China } \\
\text { National Pharmaceutical } \\
\text { Group (Sinopharm) }\end{array}$ & China & $\begin{array}{l}\text { Approved in } 15 \\
\text { countries, } \\
6 \text { trials in } 7 \\
\text { countries }\end{array}$ \\
\hline EpiVacCorona & Peptide vaccine & $\begin{array}{l}\text { Federal Budgetary Research } \\
\text { Institution State Research } \\
\text { Center of Virology and } \\
\text { Biotechnology }\end{array}$ & Russia & $\begin{array}{l}\text { Approved in } 1 \\
\text { country, } \\
2 \text { trials in } 1 \\
\text { country }\end{array}$ \\
\hline $\begin{array}{l}\text { Convidicea } \\
\text { (Ad5-nCoV) }\end{array}$ & $\begin{array}{l}\text { Recombinant } \\
\text { vaccine } \\
\text { (adenovirus type } 5 \\
\text { vector) }\end{array}$ & CanSino Biologics & China & $\begin{array}{l}\text { Approved in } 3 \\
\text { countries, } \\
6 \text { trials in } 6 \\
\text { countries }\end{array}$ \\
\hline Covaxin & $\begin{array}{l}\text { Inactivated } \\
\text { vaccine }\end{array}$ & Bharat Biotech, ICMR & India & $\begin{array}{l}\text { Approved in } 2 \\
\text { countries, } \\
5 \text { trials in } 1 \\
\text { country }\end{array}$ \\
\hline $\begin{array}{l}\text { JNJ-78436735 } \\
\text { (formerly } \\
\text { Ad26.COV2.S) }\end{array}$ & $\begin{array}{l}\text { Non-replicating } \\
\text { viral vector }\end{array}$ & $\begin{array}{l}\text { Janssen Vaccine (Johnson } \\
\text { \& Johnson) }\end{array}$ & $\begin{array}{l}\text { The } \\
\text { Netherlands, } \\
\text { USA }\end{array}$ & $\begin{array}{l}\text { Approved in } 3 \\
\text { countries } \\
6 \text { trials in } 17 \\
\text { countries, } \\
\text { (Phase } 3 \\
\text { clinical trials) }\end{array}$ \\
\hline \multicolumn{5}{|c|}{ Vaccine candidates in development } \\
\hline Candidate & Mechanism & Sponsor & Trial phase & Institution \\
\hline NVX-CoV2373 & Protein subunit & Novavax & Phase 3 & Novavax \\
\hline RBD-Dimer & $\begin{array}{l}\text { Recombinant } \\
\text { vaccine }\end{array}$ & $\begin{array}{l}\text { Anhui Zhifei Longcom } \\
\text { Biopharmaceutical, } \\
\text { Institute of Microbiology } \\
\text { of the Chinese Academy of } \\
\text { Sciences } \\
\end{array}$ & Phase 3 & Various \\
\hline ZyCoV-D & $\begin{array}{l}\text { DNA vaccine } \\
\text { (plasmid) }\end{array}$ & Zydus Cadila & Phase 2 & Zydus Cadila \\
\hline $\mathrm{CVnCoV}$ & $\begin{array}{l}\text { mRNA-based } \\
\text { vaccine }\end{array}$ & $\begin{array}{l}\text { CureVac; } \\
\text { GlaxoSmithKline } \\
\end{array}$ & Phase $2 b / 3$ & CureVac \\
\hline INO-4800 & $\begin{array}{l}\text { DNA vaccine } \\
\text { (plasmid) }\end{array}$ & Inovio Pharmaceuticals & Phase $2 / 3$ & $\begin{array}{l}\text { Center for } \\
\text { Pharmaceutical } \\
\text { Research, } \\
\text { Kansas City. } \\
\text { Mo.; University } \\
\text { of } \\
\text { Pennsylvania, } \\
\text { Philadelphia }\end{array}$ \\
\hline VIR-7831 & $\begin{array}{l}\text { Plant-based } \\
\text { adjuvant vaccine }\end{array}$ & $\begin{array}{l}\text { Medicago; } \\
\text { GlaxoSmithKline; } \\
\text { Dynavax }\end{array}$ & Phase $2 / 3$ & Medicago \\
\hline
\end{tabular}




\begin{tabular}{|c|c|c|c|c|}
\hline \multicolumn{5}{|c|}{ Vaccine candidates in development } \\
\hline Candidate & Mechanism & Sponsor & Trial phase & Institution \\
\hline hAd5-Covid-19 & $\begin{array}{l}\text { Adenovirus-based } \\
\text { vaccine }\end{array}$ & ImmunityBio; NantKwest & Phase $2 / 3$ & \\
\hline UB-612 & $\begin{array}{l}\text { Multitope peptide- } \\
\text { based vaccine }\end{array}$ & COVAXX & Phase $2 / 3$ & $\begin{array}{l}\text { United } \\
\text { Biomedical } \\
\text { Inc. (UBI) }\end{array}$ \\
\hline $\begin{array}{l}\text { Abdala } \\
\text { (CIGB 66) }\end{array}$ & $\begin{array}{l}\text { Protein subunit } \\
\text { vaccine }\end{array}$ & $\begin{array}{l}\text { Finlay Institute of } \\
\text { Vaccines }\end{array}$ & Phase 2 & $\begin{array}{l}\text { Finlay Institute } \\
\text { of Vaccines }\end{array}$ \\
\hline BNT162b1 & $\begin{array}{l}\text { mRNA-based } \\
\text { vaccine }\end{array}$ & Pfizer, BioNTech & Phase $1 / 2 / 3$ & $\begin{array}{l}\text { Multiple study } \\
\text { sites in Europe, } \\
\text { North America } \\
\text { and China }\end{array}$ \\
\hline AdCLD-CoV19 & $\begin{array}{l}\text { Adenovirus-based } \\
\text { vaccine }\end{array}$ & Cellid; LG Chem & Phase $1 / 2 \mathrm{a}$ & $\begin{array}{l}\text { Korea } \\
\text { University } \\
\text { Guro Hospital }\end{array}$ \\
\hline Nanocovax & $\begin{array}{l}\text { Recombinant } \\
\text { vaccine (Spike } \\
\text { protein) }\end{array}$ & $\begin{array}{l}\text { Nanogen } \\
\text { Biopharmaceutical }\end{array}$ & Phase $1 / 2$ & $\begin{array}{l}\text { Military } \\
\text { Medical } \\
\text { Academy } \\
\text { (Vietnam) }\end{array}$ \\
\hline EuCorVac-19 & $\begin{array}{l}\text { nanoparticle } \\
\text { vaccine }\end{array}$ & EuBiologics & Phase $1 / 2$ & $\begin{array}{l}\text { Eunpyeong } \\
\text { St. Mary's } \\
\text { Hospital }\end{array}$ \\
\hline IIBR-100 & $\begin{array}{l}\text { Recombinant } \\
\text { vesicular } \\
\text { stomatitis virus } \\
\text { (rVSV) vaccine }\end{array}$ & $\begin{array}{l}\text { Israel Institute for } \\
\text { Biological Research }\end{array}$ & Phase $1 / 2$ & $\begin{array}{l}\text { Hadassah } \\
\text { Medical } \\
\text { Center; Sheba } \\
\text { Medical Center } \\
\text { Hospital }\end{array}$ \\
\hline $\begin{array}{l}\text { Recombinant } \\
\text { (SF9 cell) }\end{array}$ & Protein subunit & $\begin{array}{l}\text { West China Hospital, } \\
\text { Sichuan University }\end{array}$ & Phase $1 / 2$ & $\begin{array}{l}\text { West China } \\
\text { Hospital, } \\
\text { Sichuan } \\
\text { University }\end{array}$ \\
\hline VLA2001 & $\begin{array}{l}\text { Inactivated } \\
\text { vaccine }\end{array}$ & $\begin{array}{l}\text { Valneva; National Institute } \\
\text { for Health Research (NIHR) }\end{array}$ & Phase $1 / 2$ & $\begin{array}{l}\text { Multiple NIHR } \\
\text { testing sites }\end{array}$ \\
\hline $\begin{array}{l}\text { No name } \\
\text { announced }\end{array}$ & $\begin{array}{l}\text { Adjuvanted } \\
\text { protein subunit } \\
\text { vaccine }\end{array}$ & CEPI & Phase $1 / 2$ & \\
\hline $\begin{array}{l}\text { AG0301- } \\
\text { COVID19 }\end{array}$ & DNA vaccine & AnGes, Inc. & Phase $1 / 2$ & $\begin{array}{l}\text { AnGes, Inc.; } \\
\text { Japan Agency } \\
\text { for Medical } \\
\text { Research and } \\
\text { Development }\end{array}$ \\
\hline BECOV2B & Protein subunit & Biologicals E limited & Phase $1 / 2$ & $\begin{array}{l}\text { Biologicals E } \\
\text { limited, India }\end{array}$ \\
\hline GX-19N & DNA vaccine & Genexine & Phase $1 / 2$ & \\
\hline $\begin{array}{l}\text { ARCT-021 } \\
\text { (LUNAR- } \\
\text { COV19) }\end{array}$ & $\begin{array}{l}\text { Self-replicating } \\
\text { RNA vaccine }\end{array}$ & $\begin{array}{l}\text { Arcturus } \\
\text { Therapeutics and Duke- } \\
\text { NUS Medical School }\end{array}$ & Phase $1 / 2$ & $\begin{array}{l}\text { Duke-NUS } \\
\text { Medical School, } \\
\text { Singapore }\end{array}$ \\
\hline
\end{tabular}




\begin{tabular}{|c|c|c|c|c|}
\hline \multicolumn{5}{|c|}{ Vaccine candidates in development } \\
\hline Candidate & Mechanism & Sponsor & Trial phase & Institution \\
\hline $\begin{array}{l}\text { No name } \\
\text { announced }\end{array}$ & $\begin{array}{l}\text { Protein subunit } \\
\text { vaccine }\end{array}$ & Sanofi; GlaxoSmithKline & Phase $1 / 2$ & Various \\
\hline AV-COVID-19 & $\begin{array}{l}\text { Dendritic cell } \\
\text { vaccine }\end{array}$ & Aivita Biomedical, Inc. & Phase $1 \mathrm{~b} / 2$ & $\begin{array}{l}\text { Rumah Sakit } \\
\text { Umum Pusat } \\
\text { Dr Kariadi }\end{array}$ \\
\hline $\begin{array}{l}\text { PTX- } \\
\text { COVID19-B }\end{array}$ & $\begin{array}{l}\text { mRNA-based } \\
\text { vaccine }\end{array}$ & $\begin{array}{l}\text { Providence Therapeutics; } \\
\text { Canadian government }\end{array}$ & Phase 1 & \\
\hline COVI-VAC & Intranasal vaccine & $\begin{array}{l}\text { Codagenix; Serum Institute } \\
\text { of India }\end{array}$ & Phase 1 & \\
\hline AKS-452 & Protein subunit & $\begin{array}{l}\text { University Medical Center } \\
\text { Groningen, Akston } \\
\text { Biosciences Corporation }\end{array}$ & Phase 2 & \\
\hline CORVax 12 & $\begin{array}{l}\text { DNA vaccine } \\
\text { (plasmid) }\end{array}$ & $\begin{array}{l}\text { OncoSec; Providence } \\
\text { Cancer Institute }\end{array}$ & Phase 1 & $\begin{array}{l}\text { Providence } \\
\text { Portland } \\
\text { Medical Center }\end{array}$ \\
\hline MVA-SARS-2-S & $\begin{array}{l}\text { Modified vaccinia } \\
\text { virus ankara } \\
\text { (MVA) vector } \\
\text { vaccine candidate }\end{array}$ & $\begin{array}{l}\text { Universitätsklinikum } \\
\text { Hamburg-Eppendorf; } \\
\text { German Center for Infection } \\
\text { Research; Philipps University } \\
\text { Marburg Medical Center; } \\
\text { Ludwig-Maximilians - } \\
\text { University of Munich } \\
\end{array}$ & Phase 1 & $\begin{array}{l}\text { University } \\
\text { Medical Center } \\
\text { Hamburg- } \\
\text { Eppendorf }\end{array}$ \\
\hline $\mathrm{COH} 04 \mathrm{~S} 1$ & $\begin{array}{l}\text { Modified vaccinia } \\
\text { virus ankara } \\
\text { (MVA) vector } \\
\text { vaccine candidate }\end{array}$ & $\begin{array}{l}\text { City of Hope Medical } \\
\text { Center; National Cancer } \\
\text { Institute }\end{array}$ & Phase 1 & $\begin{array}{l}\text { City of Hope } \\
\text { Medical Center }\end{array}$ \\
\hline CoVac-1 & $\begin{array}{l}\text { Multi-peptide } \\
\text { vaccine candidate }\end{array}$ & $\begin{array}{l}\text { University Hospital } \\
\text { Tuebingen }\end{array}$ & Phase 1 & $\begin{array}{l}\text { University } \\
\text { Hospital } \\
\text { Tuebingen }\end{array}$ \\
\hline AdimrSC-2f & $\begin{array}{l}\text { Protein subunit } \\
\text { vaccine }\end{array}$ & Adimmune & Phase 1 & Adimmune \\
\hline bacTRL-Spike & $\begin{array}{l}\text { Monovalent oral } \\
\text { vaccine } \\
\text { (bifidobacteria) }\end{array}$ & Symvivo & Phase 1 & $\begin{array}{l}\text { Symvivo } \\
\text { Corporation }\end{array}$ \\
\hline COVAX-19 & $\begin{array}{l}\text { Monovalent } \\
\text { recombinant } \\
\text { protein vaccine }\end{array}$ & Vaxine Pty Ltd. & Phase 1 & $\begin{array}{l}\text { Royal Adelaide } \\
\text { Hospital }\end{array}$ \\
\hline $\begin{array}{l}\text { DelNS1-2019- } \\
\text { nCoV-RBD- } \\
\text { OPT1 }\end{array}$ & $\begin{array}{l}\text { Replicating viral } \\
\text { vector }\end{array}$ & $\begin{array}{l}\text { Xiamen University, } \\
\text { Beijing Wantai Biological } \\
\text { Pharmacy }\end{array}$ & Phase 1 & $\begin{array}{l}\text { Jiangsu } \\
\text { Provincial } \\
\text { Centre For } \\
\text { Disease Control } \\
\text { and Prevention } \\
\end{array}$ \\
\hline GRAd-COV2 & $\begin{array}{l}\text { Adenovirus-based } \\
\text { vaccine }\end{array}$ & $\begin{array}{l}\text { ReiThera; Leukocare; Univ } \\
\text { ercells }\end{array}$ & Phase 1 & $\begin{array}{l}\text { Lazzaro } \\
\text { Spallanzani } \\
\text { National } \\
\text { Institute for } \\
\text { Infectious } \\
\text { Diseases }\end{array}$ \\
\hline
\end{tabular}




\begin{tabular}{|c|c|c|c|c|}
\hline \multicolumn{5}{|c|}{ Vaccine candidates in development } \\
\hline Candidate & Mechanism & Sponsor & Trial phase & Institution \\
\hline Sclamp & $\begin{array}{l}\text { Protein subunit } \\
\text { vaccine }\end{array}$ & $\begin{array}{l}\text { CSL; The University of } \\
\text { Queensland }\end{array}$ & Phase 1 & \\
\hline SCB-2019 & $\begin{array}{l}\text { Protein subunit } \\
\text { vaccine }\end{array}$ & $\begin{array}{l}\text { GlaxoSmithKline, Sanofi, } \\
\text { Clover } \\
\text { Biopharmaceuticals, } \\
\text { Dynavax and Xiamen } \\
\text { Innovax; CEPI }\end{array}$ & Phase 1 & $\begin{array}{l}\text { Linear Clinical } \\
\text { Research } \\
\text { (Australia) }\end{array}$ \\
\hline VXA-CoV2-1 & $\begin{array}{l}\text { Recombinant } \\
\text { vaccine } \\
\text { (adenovirus type } 5 \\
\text { vector) }\end{array}$ & Vaxart & Phase 1 & Vaxart \\
\hline AdCOVID & Intranasal vaccine & Altimmune & Phase 1 & $\begin{array}{l}\text { University of } \\
\text { Alabama at } \\
\text { Birmingham }\end{array}$ \\
\hline
\end{tabular}

BARDA: Biomedical Advanced Research and Development Authority, part of the HHS office of the assistant secretary for preparedness and response, was established to aid in securing our nation from chemical, biological, radiological, and nuclear (CBRN) threats, as well as from pandemic influenza (PI) and emerging infectious diseases (EID); NIAID: National Institute of Allergy and Infectious Diseases; ICMR: Indian Council of Medical Research; CEPI: The Coalition for Epidemic Preparedness Innovations (CEPI) is a foundation that takes donations from public, private, philanthropic, and civil society organizations, to finance independent research projects to develop vaccines against emerging infectious diseases (EID); OWS: Operation Warp Speed is a collaboration of several US government departments including Health and Human Services (HHS) and subagencies, Defense, Agriculture, Energy and Veterans Affairs and the private sector. OWS has funded JNJ-78436735 (Janssen), mRNA-1273 (Moderna), and NVX-CoV2373 (Novavax), V590 (Merck/IAVI), V591 (Merck/Themis), AZD1222 (AstraZeneca/University of Oxford), and the candidate developed by Sanofi and GlaxoSmithKline; COVAX: The COVAX initiative, part of the World Health Organization's (WHO) Access to COVID-19 Tools (ACT) Accelerator, is being spearheaded by the Coalition for Epidemic Preparedness Innovations (CEPI); Gavi, the Vaccine Alliance; and WHO. The goal is to work with vaccine manufacturers to offer low-cost COVID-19 vaccines to countries. CEPI's candidates from companies Inovio, Moderna, CureVac, Institut Pasteur/Merck/Themis, AstraZeneca/University of Oxford, Novavax, University of Hong Kong, Clover Biopharmaceuticals, and University of Queensland/CSL are part of the COVAX initiative.

Table 2: Current therapeutic trials to target SARS-CoV-2 infection

\begin{tabular}{|l|l|l|l|l|}
\hline $\begin{array}{l}\text { Study } \\
\text { identifier }\end{array}$ & Drug & Sponsor & Stage & Status \\
\hline NCT04452435 & C21 & Vicore Pharma AB & Phase 2 & Results not reported \\
\hline NCT04445272 & $\begin{array}{l}\text { Tocilizumab (anti- } \\
\text { cytokine therapy) }\end{array}$ & $\begin{array}{l}\text { Fundacion SEIMC- } \\
\text { GESIDA/ Roche } \\
\text { Pharma AG, } \\
\text { Dynamic Science } \\
\text { S.L }\end{array}$ & Phase 2 & Results not reported \\
\hline NCT04356937 & Tocilizumab & $\begin{array}{l}\text { Massachusetts } \\
\text { General Hospital/ } \\
\text { Genentech }\end{array}$ & Phase 3 & $\begin{array}{l}\text { Drug was not found } \\
\text { efficacious in preventing } \\
\text { death or intubation. }\end{array}$ \\
\hline
\end{tabular}




\begin{tabular}{|c|c|c|c|c|}
\hline $\begin{array}{l}\text { Study } \\
\text { identifier }\end{array}$ & Drug & Sponsor & Stage & Status \\
\hline NCT04646109 & Ivermectin & $\begin{array}{l}\text { Afyonkarahisar } \\
\text { Health Sciences } \\
\text { University/ NeuTec } \\
\text { Pharma }\end{array}$ & Phase 3 & \\
\hline NCT04390022 & Ivermectin & $\begin{array}{l}\text { Clinica Universidad } \\
\text { de Navarra, } \\
\text { Universidad de } \\
\text { Navarra/Barcelona } \\
\text { Institute for Global } \\
\text { Health }\end{array}$ & Phase 2 & $\begin{array}{l}\text { No efficacy observed } \\
\text { upon early administration } \\
\text { in low risk patients; } \\
\text { significant lowering of } \\
\text { viral and IgG load } \\
\text { observed. }\end{array}$ \\
\hline NCT04343092 & $\begin{array}{l}\text { Ivermectin } \\
\text { (add-on therapy) }\end{array}$ & $\begin{array}{l}\text { University of } \\
\text { Baghdad }\end{array}$ & Phase 1 & $\begin{array}{l}\text { Better efficacy and safety } \\
\text { profile of ivermectin } \\
\text { along with } \\
\text { hydroxychloroquine and } \\
\text { azithromycin }\end{array}$ \\
\hline NCT04523831 & $\begin{array}{l}\text { Ivermectin Plus } \\
\text { Doxycycline }\end{array}$ & $\begin{array}{l}\text { Dhaka Medical } \\
\text { College }\end{array}$ & Phase 3 & $\begin{array}{l}\text { Effective in mild-to- } \\
\text { moderate cases within } 72 \\
\text { h along with standard } \\
\text { supportive care, without } \\
\text { the need for } \\
\text { hospitalization. }\end{array}$ \\
\hline NCT04422561 & $\begin{array}{l}\text { Ivermectin } \\
\text { (prophylaxis) }\end{array}$ & Zagazig University & $\begin{array}{l}\text { Phase 2, } \\
\text { Phase } 3\end{array}$ & $\begin{array}{l}\text { Significantly lower } \\
\text { incidences of development } \\
\text { of disease in healthcare } \\
\text { workers when compared } \\
\text { with the control arm. }\end{array}$ \\
\hline $\begin{array}{l}\text { PMID } \\
33592050\end{array}$ & $\begin{array}{l}\text { Ivermectin } \\
\text { (prophylaxis) }\end{array}$ & AIIMS Bhubaneswar & $\begin{array}{l}\text { Epidemi } \\
\text { ological } \\
\text { (case- } \\
\text { control } \\
\text { study) }\end{array}$ & $\begin{array}{l}\text { Significantly lower } \\
\text { incidences of } \\
\text { development of disease } \\
\text { in healthcare workers } \\
\text { with two doses when } \\
\text { compared with the } \\
\text { control arm. No efficacy } \\
\text { seen with a single dose. }\end{array}$ \\
\hline NCT04542694 & Favipiravir & Promomed, LLC & Phase 3 & $\begin{array}{l}\text { Negative PCR by day } 10 \\
\text { [98 / } 100 \text { in the FV } \\
\text { group, } 79 / 100 \text { in the } \\
\text { SoC group] (P=0.00016). } \\
\text { SoC: Std. of care }\end{array}$ \\
\hline NCT04280705 & Remdesivir & $\begin{array}{l}\text { National Institute of } \\
\text { Allergy and } \\
\text { Infectious Diseases } \\
\text { (NIAID) }\end{array}$ & Phase 3 & $\begin{array}{l}\text { Remdesivir showed } \\
\text { lower recovery time ( } 10 \\
\text { days) as compared to } \\
\text { placebo (15 days). }\end{array}$ \\
\hline NCT04292730 & Remdesivir & Gilead Sciences & Phase 3 & $\begin{array}{l}\text { Moderately ill patients } \\
\text { receiving longer treatment } \\
\text { (10 days) reported no } \\
\text { difference while those } \\
\text { receiving treatment for a } \\
\text { shorter period ( } 5 \text { days) } \\
\text { showed better clinical } \\
\text { status distribution. }\end{array}$ \\
\hline
\end{tabular}




\begin{tabular}{|c|c|c|c|c|}
\hline $\begin{array}{l}\text { Study } \\
\text { identifier }\end{array}$ & Drug & Sponsor & Stage & Status \\
\hline NCT04292899 & Remdesivir & Gilead Sciences & Phase 3 & $\begin{array}{l}\text { Severely ill patients } \\
\text { receiving treatment for } 5 \\
\text { or } 10 \text { days did not show } \\
\text { any significant difference. }\end{array}$ \\
\hline NCT04329923 & Hydroxychloroquine & $\begin{array}{l}\text { Ravi Amaravadi, } \\
\text { University of } \\
\text { Pennsylvania }\end{array}$ & Phase 2 & $\begin{array}{l}\text { Study was terminated } \\
\text { because no significant } \\
\text { difference in disease } \\
\text { incidence was observed } \\
\text { between } \\
\text { hydroxychloroquine and } \\
\text { placebo. }\end{array}$ \\
\hline NCT04435808 & $\begin{array}{l}\text { Hydroxychloroquine } \\
\text { (prophylaxis) }\end{array}$ & $\begin{array}{l}\text { University of New } \\
\text { Mexico }\end{array}$ & $\begin{array}{l}\text { Phase 1, } \\
\text { Phase } 2\end{array}$ & Terminated for futility \\
\hline NCT04491994 & Hydroxychloroquine & $\begin{array}{l}\text { UNICEF/ Pak } \\
\text { Emirates Military } \\
\text { Hospital }\end{array}$ & Phase 3 & $\begin{array}{l}\text { No effect on disease } \\
\text { progression or virus } \\
\text { clearance was observed } \\
\text { when combined with } \\
\text { standard-of-care. }\end{array}$ \\
\hline NCT04491240 & $\begin{array}{l}\text { MSC-derived } \\
\text { exosome }\end{array}$ & $\begin{array}{l}\text { Samara Regional } \\
\text { Medical Center } \\
\text { Dinasty }\end{array}$ & $\begin{array}{l}\text { Phase 1, } \\
\text { Phase } 2\end{array}$ & \\
\hline $\begin{array}{l}\text { PMID } \\
32380908\end{array}$ & $\begin{array}{l}\text { MSC-derived } \\
\text { exosome }\end{array}$ & $\begin{array}{l}\text { Authors/ Christ } \\
\text { Hospital }\end{array}$ & $\begin{array}{l}\text { Non- } \\
\text { randomiz } \\
\text { ed open- } \\
\text { label } \\
\text { cohort } \\
\text { study }\end{array}$ & $\begin{array}{l}\text { Safety was established, } \\
\text { with } 83 \% \text { survival rate } \\
\text { and improvement in } \\
\text { clinical paramters. }\end{array}$ \\
\hline NCT04343261 & $\begin{array}{l}\text { Convalescent } \\
\text { Plasma }\end{array}$ & Saint Francis Care & Phase 2 & $\begin{array}{l}\text { Safety was established in } \\
\text { critical patients who were } \\
\text { administered with } \\
\text { convalescent plasma from } \\
\text { recovered donors ( } 1: 320 \\
\text { anti-SARS-CoV2 IgG } \\
\text { titre); likely to be } \\
\text { efficacious if administered } \\
\text { at an early stage. }\end{array}$ \\
\hline NCT04446429 & $\begin{array}{l}\text { Proxalutamide (anti- } \\
\text { androgen) }\end{array}$ & $\begin{array}{l}\text { Applied Biology, } \\
\text { Inc. }\end{array}$ & - & $\begin{array}{l}\text { Safety was established, } \\
\text { with no serious adverse } \\
\text { event compared to } 27 \% \\
\text { in control. }\end{array}$ \\
\hline NCT04410159 & Gargling & $\begin{array}{l}\text { Universiti Sains } \\
\text { Islam Malaysia }\end{array}$ & Phase 2 & $\begin{array}{l}\text { Gargling with } 1 \% \\
\text { povidone-iodine } \\
\text { (Betadine }^{\circledR} \text { ) and essential } \\
\text { oils (Listerine }{ }^{\circledR} \text { ) was } \\
\text { found to be efficacious in } \\
\text { management of the early } \\
\text { stage of the disease. }\end{array}$ \\
\hline NCT04331366 & $\begin{array}{l}\text { Bidirectional } \\
\text { Oxygenation Valve }\end{array}$ & Emory University & - & \\
\hline
\end{tabular}

*Only completed studies have been included (Accession date: Feb. 19, 2021) 


\section{LESSONS LEARNT}

\subsection{Ebola Virus Disease (EVD) outbreak}

Countries need to support their core infrastructure to detect and respond to public health outbreaks. Collaborative research must be carried out in partnership with affected communities and across sectors. By investing in robust health systems, it is possible to have a rational response strategy in place (Moon et al., 2017). The Ebola outbreak in West Africa in 2014-2016 is a case in point. It was observed that many frontline health workers lacked appropriate training in emergency preparedness and response, several of them worked in unsafe environment with subpar equipment. This frail system resulted in more than 800 healthcare workers being infected (Piot et al., 2019). Since then, several initiatives have been taken by WHO to advance national and global awareness. One approach was to implement the Joint External Evaluation (JEE) tool to independently assess national response mechanisms to public health threats, whether natural, accidental or intentional, as per their obligation under the International Health Regulations (2005) (Leigh et al., 2018). By mid-2019, 100 countries had already completed this voluntary external evaluation (https://www.cdc.gov/globalhealth/healthprotection/ghs/ihr/index.html; accessed on 24.02.2021). The $73^{\text {rd }}$ World Health Assembly organized by WHO in November, 2020 adopted resolution EB146.R10 to strengthen preparedness for health emergencies and commitment to better prepare for health emergencies such as COVID19, through "full" compliance with the International Health Regulations (2005) (https://www.who.int/westernpacific/about/how-we-work/programmes/who-healthemergencies-programme; accessed on 05.03.2021). Another initiative of WHO has been the Health Emergencies Programme to reinforce operational capabilities and to support countries in their preparedness against public health emergencies. The Coalition for Epidemic Preparedness Innovations (CEPI) was launched at Davos in 2017. This is a global venture with stakeholders from public, private, philanthropic and civil society organisations working together to develop vaccines against infectious diseases and to facilitate availability of such vaccines to the affected population as required. In partnership with Gavi, the Vaccine Alliance and WHO, CEPI is leading a global alliance (COVAX) with the aim of equitable distribution of 2 billion doses of vaccine by December, 2021. The Pandemic Emergency Financing Facility (PEF) set up by the World 
Bank aims to fund research to develop preventive strategies for high-severity outbreaks becoming pandemics (https://www.worldbank.org/en/topic/pandemics/brief/pandemicemergency-financing-facility; accessed on 05.03.2021).

Global initiatives need to be supplemented with local/national measures. One of the major hindrances in the development of infrastructure for health management has been sporadic and inadequate funding (Moon et al., 2017). The promises to nurture research and development in health sciences and health management die out as the public health threat passes till the next calamity strikes. The layout of developed countries is higher for both health as well as R\&D activities while it is significantly lower in the developing countries. In 2018, Germany spent $11.43 \%$ and $3.09 \%$ of its GDP on health and R\&D respectively while U.S.A. spent $16.89 \%$ and $2.84 \%$ respectively on the same activities. On the other hand, India spent a meagre $3.54 \%$ and $0.65 \%$ of its GDP on health and R\&D respectively, during this period while China spent 5.35\% and 2.19\% and Brazil spent $9.51 \%$ and $1.26 \%$ respectively. Early investment to strengthen and incentivize $R \& D$ on infectious agents is a necessity. Despite early studies on EVD in the 1970s, no approved drugs, vaccines or rapid diagnostic tests were available when the outbreak occurred in West Africa (Piot \& Spencer, 2018). Another factor is the political stability in the affected country. Although the EBV outbreak in Democratic Republic of the Congo in 2018-2020 saw a synchronized response both nationally and internationally, cases in the eastern part of the country could not be contained because of the uncertain political situation which has seen civilians and healthcare workers being targeted. In February of this year, cases of EVD were reported in Gouécké sub-prefecture of Guinea. The Ministry of Health, along with WHO and GOARN (Global Outbreak Alert and Response Network), has initiated a planned response scheme to contain the outbreak. The effort underlies the need for collaboration between different agencies, equitable access to sophisticated infrastructure and having a standard operating procedure in place for rapid and secure response.

Of concern has been the persistence of Ebola viral particles in patients long after the symptoms had disappeared in survivors. After the declaration of end of EBV transmission by WHO at the end of 2015 in Guinea, the country reported four deaths in 
the following 8-16 weeks which were diagnosed as being due to EBV infection (Diallo et al., 2016). Sequencing showed that this virus was the same as the previous one, thus eliminating the possibility of a fresh round of animal-to-human transmission. Epidemiological tracing showed that a survivor of the previous outbreak had transferred it to the current cases via seminal fluid. RT-PCR analysis showed persistence of viral RNA in the semen for 531 days. The virus survived in immunologically protected testes, and its rate of replication and rate of mutation were low (Diallo et al., 2016). Following this, a cohort study was carried out in EBV survivors in Sierra Leone (Deen et al., 2017). Detection of viral RNA was $100 \%$ in semen of survivors immediately upon discharge and decreased slowly with time. Even after 13-15 months and 16-18 months after discharge, $11 \%$ and $4 \%$ survivors, respectively, tested positive for the presence of viral RNA (Deen et al., 2017). These results suggest that long-term monitoring of survivors of viral infections, including COVID-19, needs to be an essential component of their post-discharge care. This will eliminate the possibility of viral survival in immune-protected tissues and organs.

\subsection{Genome library}

As the COVID-19 pandemic has shown, information about the viral genome is the key to the development of any treatment strategy. Genome libraries need to be set up as online databases which are freely accessible and permit rapid international sharing of viral sequencing data. This will help the international research community to collaborate and understand the disease, and develop therapeutic approaches. At present, there are two major genome data sharing platforms for COVID-19. GISAID hosts more than 4,50,000 viral genomes and the International Nucleotide Sequence Database Collaboration (INSDC) (comprising of the EMBL's European Bioinformatics Institute (EMBL-EBI), the DNA Data Bank of Japan (DDBJ) and the National Library of Medicine's National Center for Biotechnology Information (NCBI)) currently hosts 2,70,000 raw SARS-CoV-2 sequences and 55,000 consensus or assembled genomes. GISAID platform was set up about a decade back to share sequence information about the influenza virus. The same tool was used by China to share initial sequence of SARS-CoV-2. Concerns have been raised by the scientific 
community over access and availability of data in GISAID (Hendriksen et al., 2019; Van Noorden, 2021). These need to be addressed fast to accelerate research especially on coronavirus variants. Such genome databases need to be established and/or strengthened for other pathogens as well. For a newly encountered pathogenic virus, these libraries will provide a rational starting point for development of therapeutic strategy, diagnostic tools and vaccines.

\subsection{Surveillance}

WHO has recognized that lower spread of the most recent EBV outbreak in Guinea has been, in part, due to surveillance and earlier identification of patients and their contacts. Contact tracing has emerged as a useful tool in countries which have successfully controlled the spread of COVID-19. Algorithms have been developed which are capable of scanning and picking up even small outbreaks and potentially not allowing them to turn into epidemics. These networks, e.g. GPHIN (Global Public Health Information Network, a collaborative effort between Health Canada and WHO) and ProMED-mail (International Society of Infectious Diseases), scan all types of news sources for cases of disease outbreaks. ProMED is an open access tool and allows users to contribute data through the 'SUBMIT INFO' tab. Since many of the newly emerging potential pandemics exhibit symptoms common to other less severe diseases, as has been seen with COVID-19, concerns have been raised about the utility of such surveillance tools. Nevertheless, monitoring digital platforms in this age may act as 'word-of-mouth' for spread of information and may serve as a useful auxiliary tool to monitor spread of infection. The case of EBV infection via sexual transmission, described above, points to the need of continued surveillance, especially in affected areas.

\subsection{Chain for transfer of information to policymakers: Role of healthcare workers}

The need to strengthen battle preparedness of frontline healthcare workers has been evidenced during the current pandemic. This is particularly true for reaching populations in remote and rural areas. There is a need to develop and revise national guidelines, standard operating procedures and continuous training and refresher 
modules in line with the latest information and national resources. Good health is the foundation for building a sustainable society. The starting point of this activity is the primary healthcare workforce as they are the primary implementation point for any health mission. In the context of India, millions of Anganwadi and ASHA (Accredited Social Health Activists) workers provide selfless service to uphold the community healthcare framework. They are the primary contact points for the vulnerable population, the women and children. Apart from promotion of healthy practices, they are also responsible for providing information about health and vaccination programmes, nutrition, hygiene and give preliminary support and first aid. Because of their knowledge about the healthcare system and their experience and familiarity with the local population, Anganwadi and ASHA workers can be used to provide much more support to the system than what they currently do. With the right training and adequate monetary compensation, they may emerge as skilled primary healthcare providers. Such workers are indispensable for improvement of healthcare infrastructure. This workforce will obviously be valuable much beyond the current COVID-19 pandemic and need to be recognized as an essential pillar of the public healthcare system in the country.

\subsection{Drug repurposing}

Any unprecedented pandemic requires rapid response by the research community to develop vaccines and therapeutics. While the development of vaccines may take years, drug repurposing can offer disease mitigation much quicker. Drug repurposing studies present a promising and accelerated strategy to develop therapy because these drugs have known safety profiles (Gupta \& Roy, 2021). For example, in case of COVID-19, many research publications reported sets of experimentally validated drugs as potential COVID-19 therapies within a very short period of time. These have been used to develop the COVID-19 Drug and Gene Set Library (https://amp.pharm.mssm.edu/covid19/), a collection of drug and gene sets related to COVID-19 research from multiple sources (Kuleshov et al., 2020). Similar databases may be developed for other infectious diseases too. Libraries directed against conserved regions can be immediately tested against any newly emerging strain. 


\subsection{Traditional medicines}

While research continues to develop therapeutic molecules against emerging health threats, traditional medicines, e.g. Ayurveda and Chinese traditional medicine, have also shown important benefits. Most traditional medicines operate under a holistic mode; the emphasis is on curing the patient rather than the disease alone. The Ministry of AYUSH has several schemes to promote Indian traditional medicines. In the context of COVID-19, several plant products have been listed as immune boosters' and have been of use in treatment. It needs to be emphasized that SARS$\mathrm{CoV}-2$, like any other pathogen, is more infectious to individuals with low immunity. Hence, immune boosters serve an essential purpose. In many cases, there is no single component which is responsible for efficacy but a fixed combination of components. The wider acceptability of traditional medicines can be ensured by subjecting these combinations to the same rigorous testing as other therapeutic molecules.

\subsection{Response of local governments}

The response of the southern state of Kerala to COVID-19 has been appreciated far and wide, including WHO. Kerala was able to utilize the lessons learnt during disasters in the past few years to build a robust emergency response protocol. It involved the community and frontline health workers optimally for contact tracing and isolation, gathering and dispersal of information, which allowed benefits to percolate to the ground level. Utilizing the advantages of low-cost telemedicine portals like e-sanjeevani and Arogyakeralam, encouraging campaigns like 'break the chain', etc. have been some other approaches. Probably due to its previous experience, Kerala has also seen the benefits of investment in setting up a robust healthcare infrastructure, which has certainly helped the state. Importantly, Kerala has also set up centres to address the psychological vulnerability of patients and survivors, an approach which has now been adopted in many other states. The experience and infrastructure of Kerala in utilizing neighbourhood groups, as in Kudumbashree, a community network to eradicate poverty and empower women, can easily be adapted by other states without high investment (https://www.who.int/india/news/featurestories/detail/responding-to-covid-19---learnings-from-kerala; accessed on 05.03.2021). Investment in health services and R\&D mission, however, is necessary. 
Some East Asian countries have had an enviable track record in containing the spread of COVID-19. South Korea did not follow strict lockdown protocols. However, the lessons that it learnt during the previous MERS outbreak helped it to manage this epidemic without any visible stringent measures. The citizens cooperated by following social distancing norms and wearing masks as they remembered the consequences of the MERS outbreak in 2015 which infected 17,000 citizens. Contact tracing was also started early, again a fallout of the MERS lesson. There was cooperation between the government, citizens and the scientific community, with each carrying out its designated function in a professional manner. South Korea has an efficient public health insurance system; the government invested in building a robust health ecosystem so that the adversities of MERS infection were not repeated. In January 2020, when the world was slowly waking up to the reality of COVID-19, South Korea had already started preparation toward large-scale testing for SARS-CoV-2.

Taiwan is another country that showed remarkable foresight in tackling COVID-19. The commonality with South Korea was the investment in a strong healthcare system which was already in place when COVID-19 struck. It was apprehended that proximity of Taiwan to mainland China would result in Taiwan becoming a 'hotspot' of COVID-19. Taiwan reported the absence of any positive case in the community by April, 2020 and this was achieved without a lockdown (Summers et al., 2020). There were well-defined nodal points, with Taiwan CDC and Central Epidemic Command Centre in leadership roles. Travel restrictions were imposed on passengers from all high risk countries/regions as early as January, 2020 which was later extended to other travelers. Officials had legal access to travel history of their citizens on national insurance which made identification of potential cases easier. Taiwan utilized big data and informatics not only for tracing and containing the pandemic, it also kept an open communication channel to fight misinformation (Wang et al., 2020). Wearing of masks was made compulsory; contact tracing used manual and digital modes. A comparison between spread of COVID-19 in Taiwan and New Zealand, which followed almost similar measures but with vastly different results, showed that the Taiwanese success is linked to the presence of dedicated national institutions to tackle infectious diseases (Summers et al., 2020). Taiwan also set up a National Health Command Centre (NHCC) after the SARS-CoV-2 outbreak whose primary responsibility is to respond to emerging health threats. The protocols devised after 
previous outbreaks by these agencies were such that they could be adapted to any emerging infection. On the other hand, New Zealand had no centralized coordinated response and the containment protocols relied on influenza virus as a model which follows a different course than SARS-CoV-2 (Summers et al., 2020).

The upside of this preplanning was that since citizens were able to carry out their normal daily routine, their livelihood was not affected and the economy of the country remained strong and productive.

Singapore too imposed strict border control measures very early during the outbreak which allowed it to contain imported cases (Chen et al., 2020). Strict legal measures were adopted to ensure compliance with social distancing norms. Cooperation with academia, increase in bed capacity in hospitals, granting tax rebates and extension of insurance coverage to all COVID-19 patients were some other measures followed by Singapore. Almost complete lockdown measures were implemented on April 7, 2020, which has given rise to the term 'circuit breaker' (Chen et al., 2020).

\section{CONCLUSION}

Coordination and preparedness are two key words which emerge from the review above. Regions which were ready with a coordinated plan and had the infrastructure to follow it up were able to avoid the most adverse consequences of the pandemic. Strengthening of the grassroot healthcare workers and bringing them within the domain of the national healthcare system is another key take-home lesson. Clear and transparent statements and risk communication will ensure wider trust of the general public in the system. Investment in $R \& D$ and health ecosystem has to take place before the next calamity strikes. Research is a continuous process and makes incremental changes in our knowledgebase in any area. This has been made clear during the current pandemic. Prior and planned investment would have eliminated the need to divert all funds to development of therapeutic strategies, investing it in uplifting the economy. This would also have allowed the economy to remain viable and productive. Stockpiling of broad spectrum antiviral drugs and facilities for mass production of vaccines needs to be in place. This is not an individual but a collective responsibility and requires synergy between science and society. Science and society go hand in hand and one cannot move ahead without the other. 


\section{REFERENCES}

Al-Osail, A. M., \& Al-Wazzah, M. J. (2017). The history and epidemiology of Middle East respiratory syndrome corona virus. Multidisciplinary Respiratory Medicine, $12: 20$.

Chen, J. I., Yap, J. C., Hsu, L. Y., \& Teo Y. Y. (2020). COVID-19 and Singapore: From early response to circuit breaker. Annals of the Academy of Medicine, Singapore, 49:561-572.

Deen, G. F., Broutet, N., Xu, W., Knust, B., Sesay, F. R., McDonald, S. L. R., Ervin, E., Marrinan, J. E., Gaillard, P., Habib, N., Liu, H., Liu, W., Thorson, A. E., Yamba, F., Massaquoi, T. A., James, F., Ariyarajah, A., Ross, C., Bernstein, K., ... Sahr, F. (2017). Ebola RNA persistence in semen of ebola virus disease survivors - Final report. New England Journal of Medicine, 377(15), 14281437. https://doi.org/10.1056/NEJMoa1511410

Diallo, B., Sissoko, D., Loman, N. J., Bah, H. A., Bah, H., Worrell, M. C., Conde, L. S., Sacko, R., Mesfin, S., Loua, A., Kalonda, J. K., Erondu, N. A., Dahl, B. A., Handrick, S., Goodfellow, I., Meredith, L. W., Cotten, M., Jah, U., Guetiya Wadoum, R. E., Rollin, P., ... Duraffour, S. (2016). Resurgence of Ebola Virus Disease in Guinea Linked to a Survivor With Virus Persistence in Seminal Fluid for More Than 500 Days. Clinical infectious diseases: an official publication of the Infectious Diseases Society of America,63(10), 1353-1356. https://doi.org/10.1093/cid/ciw601

Ghedin, E., Sengamalay, N. A., Shumway, M., Zaborsky, J., Feldblyum, T., Subbu, V., Spiro, D. J., Sitz, J., Koo, H., Bolotov, P., Dernovoy, D., Tatusova, T., Bao, Y., St George, K., Taylor, J., Lipman, D. J., Fraser, C. M., Taubenberger, J. K., \& Salzberg, S. L. (2005). Large-scale sequencing of human influenza reveals the dynamic nature of viral genome evolution. Nature,437(7062), 1162-1166. https://doi.org/10.1038/nature04239

Gupta, M. N., \& Roy, I. (2021). Drugs, host proteins and viral proteins: How their promiscuities shape antiviral design. Biological Reviews of the Cambridge Philosophical Society, 96, 205-222. 
Hendriksen, R. S., Bortolaia, V., Tate, H., Tyson, G. H., Aarestrup, F. M., \& McDermott, P. F. (2019). Using Genomics to Track Global Antimicrobial Resistance. Frontiers in public health, 7, 242. https://doi.org/10.3389/fpubh. 2019.00242

Kaplan, E. H. (2020). Containing 2019-nCoV (Wuhan) coronavirus. Health Care Management Science, 23, 311-314.

Kuleshov, M. V., Stein, D. J., Clarke, D., Kropiwnicki, E., Jagodnik, K. M., Bartal, A., Evangelista, J. E., Hom, J., Cheng, M., Bailey, A., Zhou, A., Ferguson, L. B., Lachmann, A., \& Ma'ayan, A. (2020). The COVID-19 Drug and Gene Set Library. Patterns (New York, N.Y.), 1(6), 100090. https://doi.org/10.1016/j. patter.2020.100090

Lundstrom, K. (2020). Coronavirus pandemic: Treatment and future prevention. Future Microbiology, 15, 1507-1521.

Lauber, C., Goeman, J. J., Parquet, M., Nga, P. T., Snijder, E. J., Morita, K., \& Gorbalenya, A. E. (2013). The footprint of genome architecture in the largest genome expansion in RNA viruses. PLoS pathogens, 9(7), e1003500. https://doi.org/10.1371/journal.ppat.1003500

Leigh, J., Fitzgerald, G., Garcia, E., \& Moon, S. (2018). Global epidemics: how well can we cope? BMJ (Clinical research ed.), 362, k3254. https://doi.org/10. 1136/bmj.k3254

Masters, P. S. (2006). The molecular biology of coronaviruses. Advances in Virus Research, 66, 193-292.

Moon, S., Leigh, J., Woskie, L., Checchi, F., Dzau, V., Fallah, M., Fitzgerald, G., Garrett, L., Gostin, L., Heymann, D. L., Katz, R., Kickbusch, I., Morrison, J. S., Piot, P., Sands, P., Sridhar, D., \& Jha, A. K. (2017). Post-Ebola reforms: ample analysis, inadequate action. BMJ (Clinical research ed.), 356, j280. https://doi.org/10.1136/bmj.j280

Piot, P., Soka, M. J., \& Spencer, J. (2019). Emergent threats: lessons learnt from Ebola. International Health, 11, 334-337. 
Piot, P., \& Spencer, J. (2018). From 1976 to 2018: Reflections on early investigations into the Ebola virus. Transactions of the Royal Society of Tropical Medicine and Hygiene, 112, 527-528.

Summers, D. J., Cheng, D., Lin, P., Barnard, D., Kvalsvig, D. A., Wilson, P. N., \& Baker, P. (2020). Potential lessons from the Taiwan and New Zealand health responses to the COVID-19 pandemic. The Lancet Regional Health - Western Pacific, 4, 100044. https://doi.org/10.1016/j.lanwpc.2020.100044

Tavares, F. N., da Costa, E. V., Kew, O. M., \& da Silva, E. E. (2013). Complete genome sequence of the last representative genotype of wild indigenous poliovirus type 1, which circulated in Brazil. Genome announcements, 1(6), e00811-13. https://doi.org/10.1128/genomeA.00811-13

Van Noorden, R. (2021). Scientists call for fully open sharing of coronavirus genome data. Nature, 590, 195-196.

Wang, C. J., Ng, C. Y., \& Brook, R. H. (2020). Response to COVID-19 in Taiwan: Big data analytics, new technology, and proactive testing. JAMA, 323, 13411342.

Woo, P. C., Huang, Y., Lau, S. K., \& Yuen, K. Y. (2010). Coronavirus genomics and bioinformatics analysis. Viruses, 2(8), 1804-1820. https://doi.org/10.3390/ v2081803

Yang, Y., Peng, F., Wang, R., Yange, M., Guan, K., Jiang, T., Xu, G., Sun, J., \& Chang, C. (2020). The deadly coronaviruses: The 2003 SARS pandemic and the 2020 novel coronavirus epidemic in China. Journal of autoimmunity, 109, 102434. https://doi.org/10.1016/j.jaut.2020.102434

How to cite this article: Prasad, S., \& Roy, I. (2021). What does our experience of COVID-19 teach us? Vantage: Journal of Thematic Analysis, 2(1): 22-41.

DOI: https://doi.org/10.52253/vjta.2020.v02i01.03

(C) The Author(s) 2021.

This work is licensed under a Creative Commons Attribution 4.0 International License which permits its use, distribution and reproduction in any medium, provided the original work is cited. 